\title{
Biological studies on fresh water snails target to Schistosoma mansoni infection
}

\author{
Abdel - Hamid Z. Abdel - Hamid , * Samia M. Abd El - Wahab * Mona M. El - \\ Tonsy and Rehab M. Abdel - Megeed \\ ( From Medicinal Chemistry Department, National Research Center, * Zoology Department, \\ Faculty of Science, Al - Azhar University for Girls, Cairo, Egypt)
}

\begin{abstract}
In this study ,upon exposure of laboratory Egyptian B. alexandrina and B. glabrata snails to its homologus strain of $S$. mansoni , two different phenotype strains " susceptible and resistant " were distinguished. The aim of this study is to investigate the biological differences between the two types of Biomphalaria snails either susceptible or resistant at different ages. The investigated parameters included mortality, survival, percentage of susceptibility / resistance to parasitic infection and fecundity . The results of the preliminary exposure experiment revealed that parental snail stock was not completely susceptible and tend to be partially refractory to infection. The results also showed a strong association between the levels of egg production and susceptibility to parasite infection . Where breeding experiment revealed that , total egg number, E/M and EM values produced by resistant phenotype of both snail species were more than those produced by susceptible progeny snails. Also, it was noticed that, egg production is associated inversely proportion with snail age .
\end{abstract}

\section{Introduction}

Schistosomiasis is a serious public health problem and second most important parasitic disease ( WHO , 2004 ). More than 600 million people in 74 countries are at risk while more than 200 million were infected ( Gibodate and Bergquist, 2000 ). The schistosome parasites are transmitted by snail intermediate hosts found in water bodies like lakes, ponds, streams, rivers, irrigation canals and dams .Snails prefer lightly shaded areas, though they show considerable adaptability to light conditions . The snails may live up to $18-24$ months, unless infected with schistosomes or other parasites, where they may die earlier. Young snails reach sexual maturity in about two months and under favorable conditions can lay 10 - 15 eggs daily ( Ayad, 1974 ).

Certain environmental factors have pronounced effects on the susceptibility of snails to infection. For example , temperature affects the penetration of miracidia to snails and their development to cercaria ( Pitchford , 1981 ; Coelho \& Bezerra , 2006 ). While, Richards ( 1984 ) suggested that the susceptibility of snails to infection is hereditary character. Outcrossing and self-fertilization are common modes of reproduction in pulmonate gastropods ( Jarne and Stadler, 1995) Some species reproduce predominantly by self-fertilization, others are facultative self-fertilizers ( selfing only occurs when no mating partners are available), and others reproduce exclusively by cross-fertilization . Furthermore , Biomphalaria snails are simultaneous hermaphrodite with ability of selffertilization ; however, in the presence of partner ( when paired ) cross-fertilization is preferred ( Vidigal et al., 1998 ) . Selfing snails often have a lower fecundity than cross-fertilizing snails ( Bayomy and Joosse ,1987 ) . It is important to verify the influence of self-fertilization performance and copulation behaviour in breeding of 
both $B$. alexandrina and B. glabrata. The purpose of this study was to determine the different biological parameters of the two Biomphalaria species under laboratory conditions .

\section{Material And Methods}

\section{Maintenance of snails :}

Target snail species for Schistosoma infection ( Biomphalaria alexandrina and Biomphalaria glabrata) were obtained from the field ( Giza governorate, Egypt ). Five-liter glass aquaria were used in the experiment . To bread the snails, $500 \mathrm{ml}$ beakers and some crystallizing dishes were used . Snail conditioned water ( SCW ) was used in the aquaria and at weekly intervals, the aquaria were cleaned and their water changed. The snails were fed daily with fresh lettuce leaves supplemented with tetramin ( fish food ).

Two hundreds snail from each species were exposed individually to 10 freshly miracidia of Egyptian strain obtained from Theodur Bilharz Research Institute, Cairo , Egypt according to Langand and Morand ( 1998 ) technique .

Snails were examined for infection after 2 weeks and continued thereafter weekly up to 10 weeks post-miracidial exposure. As described by Larson et al.( 1996), any snails in which high infection frequencies were observed are considered susceptible and those uninfected are considered as resistant. Snails in which developing parasites are not evident were re - exposed individually to 10 miracidia / snail .

\section{Selfing / out crossing :}

Selection on the basis of health, size and age , methods of separating schistosome resistant and susceptible strains from both $B$. alexandrina and $B$. glabrata were performed according to the method described by Zanotti-Magalhaes et al. (1997) . Both susceptible and resistant parent stocks of either B. alexandrina or $B$. glabrata snails were reared singly for selffertilization . These self-fertilized snails were used for breading and for current studies as following :

Different developmental stages juvenile ( $\mathbf{J}$ ), adult ( Ad ) and senescent ( Se ) from either susceptible or resistant strains were obtained in successive generations by rearing snails singly each in $500 \mathrm{ml}$ plastic container containing SCW . On the other hand, crosses among adult and senescent snails were performed .

In self and cross-fertilization, snails readily deposited their eggs on a plastic sheets floating on the surface of water . Eggs of each snail group were transferred into other plastic containers contain SCW . Oviposition was measured by counting the number of egg mass and the number of eggs in each clutch. This was undertaken for a period of one week. Five replicates were performed for each group . Egg masses were incubated in containers containing SCW until hatching completely .

Newly hatched snails were calculated and transformed into petri-dishes ( 25 snails I dish ) containing mud, source of calcium carbonate ( chalk ) and SCW. The snails were noticed weekly up to 8 weeks to record the growth and maturity rates .

The results were analyzed by the ANOVA test ( Zar, 1996 ) .

\section{Results}

Susceptibility / resistance of Biomphalaria snails towards its homologous strain of $S$. mansoni.

The result pointed out that, about $36.7 \%$ \& $30.4 \%$ of the examined $B$. alexandrina and $B$. glabrata were resistant respectively, in contrast with $63.3 \%$ \& $69.6 \%$ of these snails yielded high infection frequencies as shown in figure (1) . As it was noticed, the curve representing percentages of susceptible snails within both two snail species started at $6^{\text {th }}$ week post miracidial exposure and reached the highest peaks by approximately the $8^{\text {th }}$ week. While the curve of refractory snails gradually decreased toward the $8^{\text {th }}$ week and up to $8^{\text {th }}$ post-exposure no changes in 
percentages of refractory snails occurred, and no marked differences were noticed at that time up to the $10^{\text {th }}$ week. The ratio of susceptibility / resistance within the same snail species at a time was fixed, although the number of snails could changes depending on the increase of mortality within susceptible ones ( Table 1 ).

Furthermore, data listed in Table ( 1 ) presents a dramatically decrease in cumulative numbers and percentage of refractory snails until reach $36(36.7 \%$ ) for $B$. alexandrina,31 ( $30.4 \%$ ) for $B$. glabrata and it was fixed during $9^{\text {th }} \& 10^{\text {th }}$ week post miracidial exposure.

\section{Mortality rate and survival of the host :}

The mortality and survival rates of the exposed B. alexandrina and B. glabrata snails were shown in Table ( 1 ) from which, it can be noticed that, the increase in mortality rate was associated with potent Schistosoma infection, since there was no death among snail species under normal condition ( control ). The total cumulative survival rate decrease gradually in both snail species where it recorded $73 \%$ for $B$. alexandrina and $87 \%$ for B. glabrata at $4^{\text {th }}$ week and reach $49 \%$ and $51 \%$ for both species at $9^{\text {th }}$ week post-exposure, respectively .

Egg production of cross and self fertilized parental snails :

Table (2) showed that, the production of egg masses of resistant and susceptible strains of both $B$. alexandrina and $B$. glabrata snail ranges from $7.0-9.3,7.3-$ $9.3,5.3-8.7$ and $6.3-7.67$, respectively . The recorded values of egg masses ( M / snail ) produced by either cross or self fertilization of resistant $B$. alexandrina snail group [ Rc ( 9.0 ), Rs ( 9.3 )] being greater than the corresponding of susceptible snail groups [ $\mathrm{Sc}(8.6)$, Ss (7.0) ] .The mean number of eggs per one clutch ( E/M ) of two resistant strains being slightly higher or lower than those corresponding in each snail group .

The mean number of eggs per each snail group produced in one week by cross or self- fertilized resistant B. alexandrina [ Rc ( 111.00 ), Rs ( 108.88 ) ] were greater than those of the two susceptible snail groups [ Sc ( 101.11$)$, Ss ( 86.33 )] . On the other hand, the number of eggs per adult snail produced by cross-fertilization [ Sc ( 101.11) \& Rc ( 111.00$)$ ] was slightly greater than those of corresponding self-fertilized snail groups [ Ss ( 86.30$)$ \& Rs ( 108.88 ) ] . The results showed also that the total egg number of both susceptible and resistant strains by either self or cross-fertilization decreased gradually by the age of parental snail. It was observed that, the number of eggs in parental adult snail ( 111.00 ) was greater than that produced from crossing between adult and senescent ( 88.89) which was greater than the two parental senescent snails ( 72.33 ).

Moreover, Table ( 2 ) showed gradual decrease in number of clutches by increasing snail age. Where adult parental snails produced egg clutches ( 9.00 ) was greater than that obtained by crossing between adult and senescent ( 8.33 ) which was greater than senescent stage ( 7.00$)$. Although, the ratio of E / $\mathrm{M}$ was not more affected by snail age. It was also noticed that, the values of egg masses produced by cross or self-fertilized resistant $B$. glabrata snail groups [ Rc ( 9.3$)$ \& Rs (8.0) ] being more greater than those two susceptible snail groups [ Sc ( 7.0$) \&$ Ss ( 7.3 ) ] . On the other hand, the numbers of eggs per one clutch ( $\mathrm{E} / \mathrm{M}$ ) produced by Sc \& Ss $(18.0 \& 17.0)$ is not significantly different from those produced by Rc \& Rs ( $17.3 \& 18.0$ ).

Furthermore, the mean total numbers of eggs per one week produced, either by cross or self-fertilized susceptible $B$. glabrata snail groups [ Sc ( 129.00$) \&$ Ss ( 126.00$)$ ] were lower than those produced by the two refractory snail groups [ Rc ( 161.78 ) \& Rs ( 144.00 ). It was also observed that, within resistant and susceptible adult snails, the egg production per snail produced by cross fertilization [ Sc ( 129.00$) \& \operatorname{Rc}(161.78$ ) ] were greater than those corresponding to self-fertilized snail groups [ Ss ( 126.00$) \&$ Rs (144.00)]. Also, the total number of eggs produced by 
susceptible fertilized ( $\mathrm{Sc} \& \mathrm{Ss}$ ) snail groups was lower than those produced by resistant fertilized ( Rc \& Rs ) snail groups.

In Table ( 3 ) and Fig. 2 , the total egg number produced per one week was inversely proportional to snail age in the case of cross-fertilization of both $B$. alexandrina and $B$. glabrata. Where it was recorded the largest values produced by crossing between two adult parental snails ( $104.78 \& 141.33$ ) which was greater than those produced from adult resistant and senescent susceptible cross-fertilization ( 82.67 \& 135.44 ). Moreover, total egg number obtained by crossing between adult susceptible and senescent resistant was greater ( $78.22 \& 121.33$ ) than those produced from two senescent parents ( $66.67 \& 106.67$ ).

Furthermore, in all B. glabrata snail groups either susceptible or resistant produced by cross-fertilization, their total egg number and egg number per mass was greater than those corresponding $B$ alexandrina snail groups ( Table 3 \& Fig. 2 ) .

\section{Hatchability of Biomphalaria snails eggs :}

In Table ( 4 ), the most observation was the number of hatched snails and its percentage in relation to total egg number produced by self-fertilization ( $63.8 \%$ ). It was significantly lower than those produced by cross-fertilization ( $67.5 \%)$ ). The recorded values showed that the number and percentage of newly hatched snails in relation to total egg number produced in susceptible strain of either $B$. alexandrina or B. glabrata was significantly lower ( 66 $\%$ ) than those produced in resistant snail phenotype ( $68.3 \%$ ). It was also noticed that, hatchability rate and number of snails reaching maturity in $B$. glabrata snails recorded larger values than those corresponding in B. alexandrina ( Table 5 \& Fig. 3 ) .

Growth rate of neonatal offspring derived by cross and self- fertilization of parental Biomphalaria snails :

The shell diameter of each snail progeny was measured weekly and the data were listed in tables $(6,7 \& 8)$. The results pointed out that, there was a gradual increase in the mean shell diameter of all tested groups and this increase was statistically non significant within the same snail species .

It was also noticed that, the growth rate of $B$. glabrata snails was faster than those of $B$. alexandrina especially at $4^{\text {th }}$ and up to $8^{\text {th }}$ week of experiment (Tables $7 \& 8$ ).

It was observed that, the phenotype of the parent snails, the type of fertilization and age of parent snails did not affect the growth rate of neonatal snails within the two snail species ( $B$. alexandrina and $B$. glabrata ). 
Table (1): Susceptibility/Resistance of $B$. alexandrina and $B$. glabrata parental snail population towards $S$. mansoni under laboratory conditions.

\begin{tabular}{|c|c|c|c|c|c|c|c|c|c|c|c|c|c|c|c|}
\hline \multirow{3}{*}{\multicolumn{2}{|c|}{ Snail phenotype }} & \multicolumn{14}{|c|}{ Time of examination after miracidial exposure } \\
\hline & & \multicolumn{2}{|c|}{28 days } & \multicolumn{2}{|c|}{35 days } & \multicolumn{2}{|c|}{42 days } & \multicolumn{2}{|c|}{49 days } & \multicolumn{2}{|c|}{56 days } & \multicolumn{2}{|c|}{63 days } & \multicolumn{2}{|c|}{70 days } \\
\hline & & B. $a$ & B.g & B. $a$ & B.g & B. $a$ & B. $g$ & B. $a$ & B. $g$ & B. $a$ & B.g & B. $a$ & B. $g$ & B. $a$ & B. $g$ \\
\hline $\begin{array}{l}\text { Cumulative } \\
\text { Total } \\
\text { survival }\end{array}$ & $\begin{array}{l}\mathrm{N} \\
\mathrm{O} \\
\%\end{array}$ & $\begin{array}{l}146 \\
(73)\end{array}$ & $\begin{array}{l}174 \\
(87)\end{array}$ & $\begin{array}{l}134 \\
(67)\end{array}$ & $\begin{array}{c}163 \\
(81.5)\end{array}$ & $\begin{array}{l}124 \\
(62)\end{array}$ & $\begin{array}{l}132 \\
(66)\end{array}$ & $\begin{array}{l}114 \\
(57)\end{array}$ & $\begin{array}{l}120 \\
(60)\end{array}$ & $\begin{array}{c}105 \\
(52.5)\end{array}$ & $\begin{array}{c}109 \\
(54.5)\end{array}$ & $\begin{array}{c}98 \\
(49)\end{array}$ & $\begin{array}{l}102 \\
(51)\end{array}$ & $\begin{array}{c}98 \\
(49)\end{array}$ & $\begin{array}{l}102 \\
(51)\end{array}$ \\
\hline $\begin{array}{l}\text { Cumulative } \\
\text { Total } \\
\text { susceptible }\end{array}$ & $\begin{array}{l}\mathrm{N} \\
\mathrm{o} \\
\%\end{array}$ & $\begin{array}{l}0 \\
0\end{array}$ & $\begin{array}{l}0 \\
0\end{array}$ & $\begin{array}{l}0 \\
0\end{array}$ & $\begin{array}{l}0 \\
0\end{array}$ & $\begin{array}{c}48 \\
(38.7)\end{array}$ & $\begin{array}{c}62 \\
(46.9)\end{array}$ & $\begin{array}{c}57 \\
(50)\end{array}$ & $\begin{array}{c}69 \\
(57.5)\end{array}$ & $\begin{array}{c}62 \\
(59.1)\end{array}$ & $\begin{array}{c}71 \\
(65.1)\end{array}$ & $\begin{array}{c}62 \\
(63.3)\end{array}$ & $\begin{array}{c}71 \\
(69.6)\end{array}$ & $\begin{array}{c}62 \\
(63.3)\end{array}$ & $\begin{array}{c}71 \\
(69.6)\end{array}$ \\
\hline $\begin{array}{l}\text { Cumulative } \\
\text { Total } \\
\text { refractory }\end{array}$ & $\begin{array}{l}\mathrm{N} \\
\mathrm{o} \\
\%\end{array}$ & $\begin{array}{c}146 \\
(100)\end{array}$ & $\begin{array}{c}174 \\
(100)\end{array}$ & $\begin{array}{c}134 \\
(100)\end{array}$ & $\begin{array}{c}163 \\
(100)\end{array}$ & $\begin{array}{c}76 \\
(61.3)\end{array}$ & $\begin{array}{c}70 \\
(53.1)\end{array}$ & $\begin{array}{c}57 \\
(50)\end{array}$ & $\begin{array}{c}51 \\
(42.5)\end{array}$ & $\begin{array}{c}43 \\
(40.9)\end{array}$ & $\begin{array}{c}38 \\
(34.9)\end{array}$ & $\begin{array}{c}36 \\
(36.7)\end{array}$ & $\begin{array}{c}31 \\
(30.4)\end{array}$ & $\begin{array}{c}36 \\
(36.7)\end{array}$ & $\begin{array}{c}31 \\
(30.4)\end{array}$ \\
\hline $\begin{array}{l}\text { Mortality } \\
\text { Total } \\
\text { Rate }\end{array}$ & $\begin{array}{l}\mathrm{N} \\
\mathrm{o} \\
\%\end{array}$ & $\begin{array}{c}54 \\
(27)\end{array}$ & $\begin{array}{c}26 \\
(13)\end{array}$ & $\begin{array}{c}66 \\
(33)\end{array}$ & $\begin{array}{c}37 \\
(18.5)\end{array}$ & $\begin{array}{c}76 \\
(38)\end{array}$ & $\begin{array}{c}68 \\
(34)\end{array}$ & $\begin{array}{c}86 \\
(43)\end{array}$ & $\begin{array}{c}80 \\
(40)\end{array}$ & $\begin{array}{c}95 \\
(47.5)\end{array}$ & $\begin{array}{c}91 \\
(45.5)\end{array}$ & $\begin{array}{l}102 \\
(51)\end{array}$ & $\begin{array}{c}98 \\
(49)\end{array}$ & $\begin{array}{l}102 \\
(51)\end{array}$ & $\begin{array}{c}98 \\
(49)\end{array}$ \\
\hline
\end{tabular}

Note: Groups of 200 snails (each of the same age and size) were used in each experiment.

B.a.: B. alexandrina.

B. g.: B. glabrata.

Table (2): Mean number of eggs and egg masses produced by self and cross-fertilization of both susceptible and resistant $B$. alexandrina and $B$. glabrata at different ages under laboratory conditions.

\begin{tabular}{|c|c|c|c|c|c|c|c|c|c|c|c|c|c|c|c|}
\hline \multirow{3}{*}{$\begin{array}{l}\text { Snail } \\
\text { phenotype }\end{array}$} & \multicolumn{6}{|c|}{ Self-fertilization } & \multicolumn{9}{|c|}{ Cross-fertilization } \\
\hline & \multicolumn{3}{|c|}{ Adult } & \multicolumn{3}{|c|}{ Senescent } & \multicolumn{3}{|c|}{ Ad \& Ad } & \multicolumn{3}{|c|}{ Ad \& Se } & \multicolumn{3}{|c|}{ Se \& Se } \\
\hline & EM & $\mathbf{E} / \mathbf{M}$ & $\mathbf{E}$ & EM & E/M & $\mathbf{E}$ & EM & E/M & $\mathbf{E}$ & EM & $\mathbf{E} / \mathbf{M}$ & $\mathbf{E}$ & EM & E/M & $\mathbf{E}$ \\
\hline $\begin{array}{l}B . \\
\text { alexandrin } \\
\text { a } \text { Resistant }\end{array}$ & $\begin{array}{c}9.33 \\
\pm \\
2.00\end{array}$ & $\begin{array}{c}11.67 \\
\pm \\
1.15\end{array}$ & 108.88 & $\begin{array}{c}8.33 \\
\pm \\
0.58\end{array}$ & $\begin{array}{c}12.00 \\
\pm \\
1.00\end{array}$ & 100.00 & $\begin{array}{c}9.00 \\
\pm \\
1.00\end{array}$ & $\begin{array}{c}12.33 \\
\pm \\
1.53\end{array}$ & 111.00 & $\begin{array}{c}8.33 \\
\pm \\
0.58\end{array}$ & $\begin{array}{c}10.67 \\
\pm \\
1.15\end{array}$ & 88.89 & $\begin{array}{c}7.00 \\
\pm \\
1.00\end{array}$ & $\begin{array}{c}10.30 \\
\pm \\
0.58\end{array}$ & $\begin{array}{l}72 . \\
33\end{array}$ \\
\hline $\begin{array}{l}B . \\
\text { alexandrina } \\
\text { Susceptibl } \\
\text { e }\end{array}$ & $\begin{array}{c}7.00 \\
\pm \\
1.00\end{array}$ & $\begin{array}{c}12.33 \\
\pm \\
1.15\end{array}$ & 86.33 & $\begin{array}{c}6.00 \\
\pm \\
1.00\end{array}$ & $\begin{array}{c}11.00 \\
\pm \\
1.00\end{array}$ & 66.00 & $\begin{array}{c}8.67 \\
\pm \\
1.52\end{array}$ & $\begin{array}{c}11.67 \\
\pm \\
1.53\end{array}$ & 101.11 & $\begin{array}{c}6.33 \\
\pm \\
0.58\end{array}$ & $\begin{array}{c}13.33 \\
\pm \\
0.58\end{array}$ & 84.44 & $\begin{array}{c}5.3 \\
\pm \\
0.58\end{array}$ & $\begin{array}{c}12.00 \\
\pm \\
1.00\end{array}$ & $\begin{array}{l}64 . \\
00\end{array}$ \\
\hline $\begin{array}{l}\text { B. } \\
\text { glabrata } \\
\text { Resistant }\end{array}$ & $\begin{array}{c}8.00 \\
\pm \\
1.53\end{array}$ & $\begin{array}{c}18.00 \\
\pm \\
1.00\end{array}$ & 144.00 & $\begin{array}{c}7.33 \\
\pm \\
0.58\end{array}$ & $\begin{array}{c}17.00 \\
\pm \\
1.00\end{array}$ & 124.67 & $\begin{array}{c}9.30 \\
\pm \\
1.52\end{array}$ & $\begin{array}{c}17.33 \\
\pm \\
1.50\end{array}$ & 161.78 & $\begin{array}{c}8.67 \\
\pm \\
1.53\end{array}$ & $\begin{array}{c}17.33 \\
\pm \\
0.58\end{array}$ & 150.22 & $\begin{array}{c}8.00 \\
\pm \\
1.00\end{array}$ & $\begin{array}{c}16.33 \\
\pm \\
0.58\end{array}$ & $\begin{array}{c}13 \\
0.6 \\
7 \\
\end{array}$ \\
\hline $\begin{array}{l}B . \\
\text { glabrata } \\
\text { Susceptible }\end{array}$ & $\begin{array}{c}7.33 \\
\pm \\
0.53\end{array}$ & $\begin{array}{c}17.00 \\
\pm \\
0.50\end{array}$ & 126.00 & $\begin{array}{c}6.67 \\
\pm \\
0.58\end{array}$ & $\begin{array}{c}16.67 \\
\pm \\
0.57\end{array}$ & 111.10 & $\begin{array}{c}7.00 \\
\pm \\
1.00\end{array}$ & $\begin{array}{c}18.00 \\
\pm \\
1.70\end{array}$ & 129.00 & $\begin{array}{c}7.67 \\
\pm \\
0.58\end{array}$ & $\begin{array}{c}17.33 \\
\pm \\
1.58\end{array}$ & 132.89 & $\begin{array}{c}6.33 \\
\pm \\
0.58\end{array}$ & $\begin{array}{c}16.33 \\
\pm \\
0.57\end{array}$ & $\begin{array}{c}10 \\
3.4 \\
4\end{array}$ \\
\hline Anova & N.S & $\begin{array}{c}\mathrm{P}< \\
0.001\end{array}$ & & N.S & $\begin{array}{c}\mathrm{P}< \\
0.001\end{array}$ & & N.S & $\begin{array}{c}\mathrm{P}< \\
0.001\end{array}$ & & N.S & $\begin{array}{c}\mathrm{P}< \\
0.001 \\
\end{array}$ & & N.S & $\begin{array}{c}\mathrm{P}< \\
0.001\end{array}$ & \\
\hline
\end{tabular}

$\mathrm{E}=$ Mean total number of eggs $\mathrm{E} / \mathrm{M}=$ Number of eggs per egg mass.EM = Number of egg masses Ad $=$ Adult 
Table (3): Mean number of eggs and egg masses produced by cross-fertilization between susceptible and resistant strains of both $B$. alexandrina and $B$. glabrata at different ages under laboratory conditions.

\begin{tabular}{|c|c|c|c|c|c|c|}
\hline \multirow{2}{*}{$\begin{array}{c}\text { Snail } \\
\text { phenotype }\end{array}$} & \multicolumn{3}{|c|}{ B. alexandrina } & \multicolumn{3}{|c|}{ B. glabrata } \\
\hline & $\begin{array}{c}\text { EM } \\
\text { Mean } \pm \text { S.D. }\end{array}$ & $\begin{array}{c}\text { E/M } \\
\text { Mean } \pm \text { S.D }\end{array}$ & $\mathbf{E}$ & $\begin{array}{c}\text { EM } \\
\text { Mean } \pm \text { S.D. }\end{array}$ & $\begin{array}{c}\text { E/M } \\
\text { Mean } \pm \text { S.D }\end{array}$ & $\mathbf{E}$ \\
\hline $\mathrm{Ad}_{\mathrm{S}} \& \mathrm{Ad}_{\mathrm{R}}$ & $7.67 \pm 1.52$ & $13.67 \pm 1.52$ & 104.78 & $8.00 \pm 1.00$ & $17.67 \pm 0.58$ & 141.33 \\
\hline $\mathrm{Se}_{\mathrm{S}} \& \mathrm{Se}_{\mathrm{R}}$ & $6.67 \pm 1.15$ & $10.00 \pm 1.00$ & 66.67 & $6.67 \pm 1.15$ & $16.00 \pm 1.00$ & 106.67 \\
\hline $\mathrm{Se}_{\mathrm{R}} \& \mathrm{Ad}_{\mathrm{S}}$ & $7.33 \pm 1.15$ & $10.67 \pm 1.53$ & 78.22 & $7.00 \pm 1.00$ & $17.33 \pm 0.58$ & 121.33 \\
\hline $\mathrm{Ad}_{\mathrm{R}} \& \mathrm{Se}_{\mathrm{S}}$ & $8.00 \pm 1.00$ & $10.33 \pm 1.15$ & 82.67 & $7.67 \pm 1.53$ & $17.67 \pm 1.15$ & 135.44 \\
\hline Anova & N.S. & $\mathrm{P}<0.001$ & & N.S. & $\mathrm{P}<0.001$ & \\
\hline
\end{tabular}

Table (4): Total number of eggs, hatching offspring and offspring reaching maturity and their percentage produced by self and cross fertilization of either susceptible or resistant B. alexandrina and $B$. glabrata at different ages.

\begin{tabular}{|c|c|c|c|c|c|c|c|c|c|c|c|c|c|c|c|}
\hline \multirow{3}{*}{$\begin{array}{c}\text { Snail } \\
\text { phenoty } \\
\text { pe }\end{array}$} & \multicolumn{6}{|c|}{ Self-fertilization } & \multicolumn{9}{|c|}{ Cross-fertilization } \\
\hline & \multicolumn{3}{|c|}{ Ad } & \multicolumn{3}{|c|}{$\mathrm{Se}$} & \multicolumn{3}{|c|}{ Ad \& Ad } & \multicolumn{3}{|c|}{ Ad \& Se } & \multicolumn{3}{|c|}{$\mathrm{Se} \& \mathrm{Se}$} \\
\hline & $\mathbf{E}$ & $\begin{array}{c}\mathrm{H} \\
(\mathrm{M} \pm \mathrm{S} \\
\mathrm{D})\end{array}$ & $\begin{array}{c}\text { RM } \\
(\mathrm{M} \pm S \\
\text { D) }\end{array}$ & $E$ & $\begin{array}{c}H \\
(M \pm S \\
D)\end{array}$ & $\begin{array}{c}\text { RM } \\
(M \pm S \\
D)\end{array}$ & $\mathbf{E}$ & $\begin{array}{c}\mathrm{H} \\
(\mathrm{M} \pm \mathrm{S} \\
\mathrm{D})\end{array}$ & $\begin{array}{c}R M \\
(M \pm S \\
D)\end{array}$ & $\mathbf{E}$ & $\begin{array}{c}\mathrm{H} \\
(\mathrm{M} \pm \mathrm{S} \\
\mathrm{D})\end{array}$ & $\begin{array}{c}\text { RM } \\
(M \pm S \\
\text { D) }\end{array}$ & $\mathbf{E}$ & $\begin{array}{c}H \\
(M \pm S \\
D)\end{array}$ & $\begin{array}{c}\text { RM } \\
(\mathrm{M} \pm S D)\end{array}$ \\
\hline $\begin{array}{l}\text { B. } \\
\text { alexandri } \\
\text { na } \\
\text { Resistant }\end{array}$ & $\begin{array}{c}108.8 \\
8\end{array}$ & $\begin{array}{c}69.30 \\
\pm \\
0.40 \\
(63.8 \% \\
)\end{array}$ & $\begin{array}{c}23.30 \\
\pm \\
2.50 \\
(34.6 \%)\end{array}$ & $\begin{array}{c}100 . \\
0\end{array}$ & $\begin{array}{c}61.80 \\
\pm \\
7.00 \\
(61.8 \%)\end{array}$ & $\begin{array}{c}20.00 \\
\pm \\
3.00 \\
(33.3 \%)\end{array}$ & $\begin{array}{c}111.0 \\
0\end{array}$ & $\begin{array}{c}7.50 \\
\pm \\
9.20 \\
(67.5 \%)\end{array}$ & $\begin{array}{c}20.33 \\
\pm \\
3.50 \\
(27 \%)\end{array}$ & $\begin{array}{c}88.8 \\
9\end{array}$ & $\begin{array}{c}59.33 \\
\pm \\
4.00 \\
(66.7 \%)\end{array}$ & $\begin{array}{c}17.33 \\
\pm \\
2.00 \\
(29.2 \%)\end{array}$ & 72.33 & $\begin{array}{c}44.33 \\
\pm \\
1.50 \\
(61.1 \%)\end{array}$ & $\begin{array}{c}14.67 \\
\pm \\
1.50 \\
(33.0 \%)\end{array}$ \\
\hline $\begin{array}{l}\text { B. } \\
\text { alexandri } \\
\text { na } \\
\text { Susceptib } \\
\text { le }\end{array}$ & 86.30 & $\begin{array}{c}54.50 \\
\pm \\
5.00 \\
(62.5 \% \\
)\end{array}$ & $\begin{array}{c}16.30 \\
\pm \\
2.50 \\
(31.3 \%)\end{array}$ & 66.0 & $\begin{array}{c}40.67 \\
\pm \\
6.00 \\
(61.6 \%)\end{array}$ & $\begin{array}{c}11.60 \\
\pm \\
2.00 \\
(28.5 \%)\end{array}$ & $\begin{array}{c}101.1 \\
1\end{array}$ & $\begin{array}{c}68.33 \\
\pm \\
8.50 \\
(67.2 \%)\end{array}$ & $\begin{array}{c}20.3 \\
\pm \\
3.00 \\
(29.7 \%)\end{array}$ & $\begin{array}{c}84.4 \\
4\end{array}$ & $\begin{array}{c}49.67 \\
\pm \\
4.50 \\
(58.8 \%)\end{array}$ & $\begin{array}{c}17.33 \\
\pm \\
1.50 \\
(34.8 \%)\end{array}$ & 64.00 & $\begin{array}{c}38.0 \\
\pm \\
3.20 \\
(59.3 \%)\end{array}$ & $\begin{array}{c}13.33 \\
\pm \\
1.50 \\
(35.0 \%)\end{array}$ \\
\hline $\begin{array}{l}\text { B. } \\
\text { glabrata } \\
\text { Resistant }\end{array}$ & $\begin{array}{c}144.0 \\
0\end{array}$ & $\begin{array}{c}98.30 \\
\pm \\
7.60 \\
(68.3 \% \\
)\end{array}$ & $\begin{array}{c}23.67 \\
\pm \\
1.50 \\
(24 \%)\end{array}$ & $\begin{array}{c}124 . \\
7\end{array}$ & $\begin{array}{c}76.67 \\
\pm \\
7.60 \\
(61.4 \%)\end{array}$ & $\begin{array}{c}21.00 \\
\pm \\
2.00 \\
(27.3 \%)\end{array}$ & $\begin{array}{c}161.7 \\
8\end{array}$ & $\begin{array}{c}117.67 \\
\pm \\
9.30 \\
(72.7 \%)\end{array}$ & $\begin{array}{c}27.00 \\
\pm \\
2.00 \\
(22.9 \%)\end{array}$ & $\begin{array}{c}150 . \\
2\end{array}$ & $\begin{array}{c}106.67 \\
\pm \\
12.58 \\
(71.0 \%)\end{array}$ & $\begin{array}{c}25.0 \\
\pm \\
1.50 \\
(23.4 \%)\end{array}$ & $\begin{array}{c}130.7 \\
0\end{array}$ & $\begin{array}{c}93.30 \\
\pm \\
6.50 \\
(69.1 \%)\end{array}$ & $\begin{array}{c}23.67 \\
\pm \\
1.50 \\
(25.0 \%)\end{array}$ \\
\hline $\begin{array}{l}\text { B. } \\
\text { glabrata } \\
\text { Susceptib } \\
\text { le }\end{array}$ & $\begin{array}{c}126.0 \\
0\end{array}$ & $\begin{array}{c}85.67 \\
\pm \\
7.00 \\
(66 \%)\end{array}$ & $\begin{array}{c}20.30 \\
\pm \\
1.50 \\
(23.6 \%)\end{array}$ & $\begin{array}{c}111 . \\
1\end{array}$ & $\begin{array}{c}65.0 \\
\pm \\
5.60 \\
(58.5 \%)\end{array}$ & $\begin{array}{c}19.00 \\
\pm \\
1.70 \\
(29.2 \%)\end{array}$ & $\begin{array}{c}129.0 \\
0\end{array}$ & $\begin{array}{c}87.00 \\
\pm \\
8.50 \\
(69.0 \%)\end{array}$ & $\begin{array}{c}25.67 \\
\pm \\
5.20 \\
(29.5 \%)\end{array}$ & $\begin{array}{c}132 . \\
9\end{array}$ & $\begin{array}{c}91.67 \\
\pm \\
9.60 \\
(68.9 \%)\end{array}$ & $\begin{array}{c}18.70 \\
\pm \\
1.50 \\
(20.4 \%)\end{array}$ & $\begin{array}{c}103.4 \\
4\end{array}$ & $\begin{array}{c}68.00 \\
\pm \\
4.60 \\
(65.7 \%)\end{array}$ & $\begin{array}{c}14.33 \\
\pm \\
1.50 \\
(21.0 \%)\end{array}$ \\
\hline
\end{tabular}

$\mathrm{E}=$ Mean total number of eggs (Mean of Egg masses X No. of eggs / mass).

$\mathrm{H}=$ Number of hatching snails.

$\mathrm{RM}=$ Number of new offspring reaching maturity . 
Table (5): Total number of eggs, hatching offspring and offspring reaching maturity and their percentage in relation to total egg number produced by cross fertilization between susceptible and resistant strains of eithher B. alexandrina or B. glabrata at different ages.

\begin{tabular}{|l|c|c|c|c|c|c|}
\hline \multirow{2}{*}{$\begin{array}{l}\text { Snail } \\
\text { phenotype }\end{array}$} & $\mathbf{E}$ & $\mathbf{H}(\mathbf{M} \pm$ SD) & $\mathbf{R}(\mathbf{M} \pm$ SD) & $\mathbf{E}$ & H $(\mathbf{M} \pm$ SD $)$ & R $(\mathbf{M} \pm$ SD) \\
\cline { 2 - 7 } & 104.78 & $65.67 \pm 7.00$ & $19.33 \pm 1.52$ & 135.44 & $91.67 \pm 3.50$ & $25.00 \pm 2.60$ \\
& $(62.6 \%)$ & $(29.4 \%)$ & & $(67.7 \%)$ & $(27.2 \%)$ \\
\hline & & $49.00 \pm 6.25$ & $13.67 \pm 1.50$ & 106.67 & $69.67 \pm 10.00$ & $16.67 \pm 1.50$ \\
& $(73.4 \%)$ & $(27.9 \%)$ & & $(65.3 \%)$ & $(23.9 \%)$ \\
\hline $\mathrm{Se}_{\mathrm{S}} \& \mathrm{Ad}_{\mathrm{R}}$ & 66.67 & $48.67 \pm 4.00$ & $15.00 \pm 1.00$ & 121.33 & $86.00 \pm 3.60$ & $17.00 \pm 2.00$ \\
& & $(62.2 \%)$ & $(30.8 \%)$ & & $(70.8 \%)$ & $(19.7 \%)$ \\
\hline $\mathrm{Se}_{\mathrm{R}} \& \mathrm{Ad}_{\mathrm{S}}$ & 78.22 & $50.00 \pm 6.00$ & $16.33 \pm 1.50$ & 141.33 & $98.67 \pm 5.50$ & $16.33 \pm 0.58$ \\
& & $(60.5 \%)$ & $(32.7 \%)$ & & $(69.8 \%)$ & $(16.5 \%)$ \\
\hline $\mathrm{Ad}_{\mathrm{R}} \& \mathrm{Se}_{\mathrm{S}}$ & 82.67 & & & & \\
\end{tabular}

$\mathrm{E}=$ Total number of eggs (Mean of egg masses $\mathrm{x}$ No. of eggs $/$ mass).

$\mathrm{H}=$ Number of hatchling snails .

$\mathrm{R}=$ Number of new offspring reaching maturity.

Table (6): Growth rate of neonatal offspring derived by self and cross fertilization of $B$. alexandrina at different ages.

\begin{tabular}{|c|c|c|c|c|c|c|c|c|c|c|c|c|c|c|c|c|c|}
\hline \multirow{2}{*}{\multicolumn{2}{|c|}{$\begin{array}{c}\text { Snail } \\
\text { phenotype }\end{array}$}} & \multicolumn{16}{|c|}{ Time of Examination / week } \\
\hline & & \multicolumn{8}{|c|}{ B. alexandrina resistant } & \multicolumn{8}{|c|}{ B. alexandrina susceptible } \\
\hline \multirow{3}{*}{$\begin{array}{l}\text { Self- } \\
\text { fertilizati } \\
\text { on }\end{array}$} & & $\begin{array}{c}\mathbf{1}^{\text {st }} \\
(\mathrm{M} \pm \mathrm{S} \\
\mathrm{D})\end{array}$ & $\begin{array}{c}2^{\text {nd }} \\
(M \pm S \\
D)\end{array}$ & $\begin{array}{c}3^{\text {rd }} \\
(M \pm S \\
D\end{array}$ & $\begin{array}{c}4^{\text {th }} \\
(M \pm S \\
D)\end{array}$ & $\begin{array}{c}5^{\text {th }} \\
(\mathrm{M} \pm \mathrm{S} \\
\mathrm{D})\end{array}$ & $\begin{array}{c}\mathbf{6}^{\text {th }} \\
(\mathbf{M} \pm \mathbf{S} \\
\text { D }\end{array}$ & $\begin{array}{c}7^{\text {th }} \\
(M \pm S \\
D\end{array}$ & $\begin{array}{c}8^{\text {th }} \\
(M \pm S \\
D\end{array}$ & $\begin{array}{c}1^{\text {st }} \\
(M \pm S \\
D\end{array}$ & $\begin{array}{c}2^{\text {nd }} \\
(M \pm S \\
D\end{array}$ & $\begin{array}{c}3^{\text {rd }} \\
(M \pm S \\
D\end{array}$ & $\begin{array}{c}4^{\text {th }} \\
(M \pm S \\
D\end{array}$ & $\begin{array}{c}5^{\text {th }} \\
(M \pm S \\
D\end{array}$ & $\begin{array}{c}\mathbf{6}^{\text {th }} \\
(\mathbf{M} \pm \mathbf{S} \\
D\end{array}$ & $\begin{array}{c}7^{\text {th }} \\
(M \pm S \\
D\end{array}$ & $\begin{array}{c}8^{\text {th }} \\
(M \pm S \\
D\end{array}$ \\
\hline & Ad & $\begin{array}{l}1.26 \\
\pm \\
0.15\end{array}$ & $\begin{array}{l}2.13 \\
\pm \\
1.53\end{array}$ & $\begin{array}{l}2.70 \\
\pm \\
0.20 \\
\end{array}$ & $\begin{array}{l}3.47 \\
\pm \\
1.53 \\
\end{array}$ & $\begin{array}{l}4.06 \\
\pm \\
0.20\end{array}$ & $\begin{array}{l}4.77 \\
\pm \\
0.15\end{array}$ & $\begin{array}{l}5.93 \\
\pm \\
0.25 \\
\end{array}$ & $\begin{array}{l}6.90 \\
\pm \\
0.40 \\
\end{array}$ & $\begin{array}{l}1.20 \\
\pm \\
0 . .20\end{array}$ & $\begin{array}{l}2.06 \\
\pm \\
0.15\end{array}$ & $\begin{array}{l}2.58 \\
\pm \\
.1 .2 \\
\end{array}$ & $\begin{array}{l}3.33 \\
\pm \\
1.53 \\
\end{array}$ & $\begin{array}{l}3.96 \\
\pm \\
1.53 \\
\end{array}$ & $\begin{array}{l}4.67 \\
\pm \\
0.15\end{array}$ & $\begin{array}{l}5.80 \\
\pm \\
0.25 \\
\end{array}$ & $\begin{array}{l}6.90 \\
\pm \\
0.30\end{array}$ \\
\hline & Se. & $\begin{array}{l}1.18 \\
\pm \\
0.15\end{array}$ & $\begin{array}{l}2.0 \\
\pm \\
0.20\end{array}$ & $\begin{array}{l}2.55 \\
\pm \\
0.16\end{array}$ & $\begin{array}{l}3.27 \\
\pm \\
0.15\end{array}$ & $\begin{array}{l}3.83 \\
\pm \\
0.13\end{array}$ & $\begin{array}{l}4.60 \\
\pm \\
0.30\end{array}$ & $\begin{array}{l}5.67 \\
\pm \\
0.35\end{array}$ & $\begin{array}{l}6.77 \\
\pm \\
0.35\end{array}$ & $\begin{array}{l}1.10 \\
\pm \\
0.20\end{array}$ & $\begin{array}{l}2.1 \\
\pm \\
0.10\end{array}$ & $\begin{array}{l}2.27 \\
\pm \\
0.25\end{array}$ & $\begin{array}{l}3.27 \\
\pm \\
0.15\end{array}$ & $\begin{array}{l}3.70 \\
\pm \\
0.20\end{array}$ & $\begin{array}{l}4.60 \\
\pm \\
0.20\end{array}$ & $\begin{array}{l}5.53 \\
\pm \\
0.47\end{array}$ & $\begin{array}{l}6.70 \\
\pm \\
0.40\end{array}$ \\
\hline \multirow{3}{*}{$\begin{array}{l}\text { Cross- } \\
\text { fertilizati } \\
\text { on }\end{array}$} & $\begin{array}{l}\text { Ad } \\
\& \\
\text { Ad }\end{array}$ & $\begin{array}{l}1.123 \\
\pm \\
0.15\end{array}$ & $\begin{array}{l}2.06 \\
\pm \\
0.20\end{array}$ & $\begin{array}{l}2.73 \\
\pm \\
0.15\end{array}$ & $\begin{array}{l}3.633 \\
\pm \\
0.15\end{array}$ & $\begin{array}{l}4.00 \\
\pm \\
0.15\end{array}$ & $\begin{array}{l}4.77 \\
\pm \\
0.25\end{array}$ & $\begin{array}{l}5.77 \\
\pm \\
0.35\end{array}$ & $\begin{array}{l}6.83 \\
\pm \\
0.30\end{array}$ & $\begin{array}{l}1.18 \\
\pm \\
0.15\end{array}$ & $\begin{array}{l}2.18 \\
\pm \\
0.16\end{array}$ & $\begin{array}{l}2.63 \\
\pm \\
0.25\end{array}$ & $\begin{array}{l}3.36 \\
\pm \\
0.15\end{array}$ & $\begin{array}{l}3.80 \\
\pm \\
0.25\end{array}$ & $\begin{array}{l}4.67 \\
\pm \\
0.25\end{array}$ & $\begin{array}{l}5.77 \\
\pm \\
0.25\end{array}$ & $\begin{array}{l}6.80 \\
\pm \\
0.40\end{array}$ \\
\hline & $\begin{array}{l}\mathrm{Se} \\
\& \\
\mathrm{Ad}\end{array}$ & $\begin{array}{l}1.230 \\
\pm \\
0.15\end{array}$ & $\begin{array}{l}2.00 \\
\pm \\
0.20\end{array}$ & $\begin{array}{l}2.70 \\
\pm \\
0.15\end{array}$ & $\begin{array}{l}3.60 \\
\pm \\
0.10\end{array}$ & $\begin{array}{l}4.00 \\
\pm \\
0.20\end{array}$ & $\begin{array}{l}4.70 \\
\pm \\
0.30\end{array}$ & $\begin{array}{l}5.77 \\
\pm \\
0.20\end{array}$ & $\begin{array}{l}6.79 \\
\pm \\
0.25\end{array}$ & $\begin{array}{l}1.17 \\
\pm \\
0.15\end{array}$ & $\begin{array}{l}2.30 \\
\pm \\
0.26\end{array}$ & $\begin{array}{l}2.60 \\
\pm \\
0.20\end{array}$ & $\begin{array}{l}3.33 \\
\pm \\
0.15\end{array}$ & $\begin{array}{l}3.70 \\
\pm \\
0.26\end{array}$ & $\begin{array}{l}4.63 \\
\pm \\
0.20\end{array}$ & $\begin{array}{l}5.70 \\
\pm \\
0.20\end{array}$ & $\begin{array}{l}6.77 \\
\pm \\
0.40\end{array}$ \\
\hline & $\begin{array}{l}\mathrm{Se} \\
\& \\
\mathrm{Se}\end{array}$ & $\begin{array}{l}1.16 \\
\pm \\
0.15\end{array}$ & $\begin{array}{l}2.0 \\
\pm \\
0 . .20\end{array}$ & $\begin{array}{l}2.70 \\
\pm \\
0.16 \\
\end{array}$ & $\begin{array}{l}3.53 \\
\pm \\
0.15\end{array}$ & $\begin{array}{l}3.93 \\
\pm \\
0.13\end{array}$ & $\begin{array}{l}4.70 \\
\pm \\
0 . .30\end{array}$ & $\begin{array}{c}5.73 \\
\pm \\
0.35\end{array}$ & $\begin{array}{l}6.87 \\
\pm \\
0.35 \\
\end{array}$ & $\begin{array}{l}1.10 \\
\pm \\
0.20\end{array}$ & $\begin{array}{l}2.10 \\
\pm \\
0.1\end{array}$ & $\begin{array}{l}2.57 \\
\pm \\
0.25\end{array}$ & $\begin{array}{l}3.27 \\
\pm \\
0.15\end{array}$ & $\begin{array}{l}3.70 \\
\pm \\
0.20\end{array}$ & $\begin{array}{l}4.60 \\
\pm \\
0.20\end{array}$ & $\begin{array}{l}5.53 \\
\pm \\
0.47\end{array}$ & $\begin{array}{l}6.70 \\
\pm \\
0.40\end{array}$ \\
\hline Anova & N.S. & N.S. & N.S. & N.S. & N.S. & N.S. & N.S. & N.S. & N.S. & N.S. & N.S. & N.S. & N.S. & N.S. & N.S. & N.S. & N.S. \\
\hline
\end{tabular}

N.S.: Non-significant . 
Table (7): Growth rate of neonatal offspring derived by self and cross-fertilization of $B$. glabrata at different ages.

\begin{tabular}{|c|c|c|c|c|c|c|c|c|c|c|c|c|c|c|c|c|c|}
\hline \multirow{2}{*}{\multicolumn{2}{|c|}{$\begin{array}{c}\text { Snail } \\
\text { phenotype }\end{array}$}} & \multicolumn{16}{|c|}{ Time of Examination / week } \\
\hline & & \multicolumn{8}{|c|}{ B. glabrata resistant } & \multicolumn{8}{|c|}{ B. glabrata susceptible } \\
\hline \multirow{3}{*}{$\begin{array}{l}\text { Self- } \\
\text { fertiliz } \\
\text { ation }\end{array}$} & & $\begin{array}{c}1^{\text {st }} \\
(\mathrm{M} \pm \mathrm{S} \\
\mathrm{D}) \\
\end{array}$ & $\begin{array}{c}2^{\text {nd }} \\
(\mathrm{M} \pm \mathrm{S} \\
\mathrm{D})\end{array}$ & $\begin{array}{c}3^{\text {rd }} \\
(\mathrm{M} \pm \mathrm{S} \\
\mathrm{D}) \\
\end{array}$ & $\begin{array}{c}4^{\text {th }} \\
(\mathrm{M} \pm \mathrm{S} \\
\mathrm{D}) \\
\end{array}$ & $\begin{array}{c}5^{\text {th }} \\
(\mathbf{M} \pm S \\
D) \\
\end{array}$ & $\begin{array}{c}\text { 6 }^{\text {th }} \\
(\mathrm{M} \pm \mathbf{S} \\
\mathrm{D}) \\
\end{array}$ & $\begin{array}{c}7^{\text {th }} \\
(M \pm S \\
D \\
\end{array}$ & $\begin{array}{c}8^{\text {th }} \\
(\mathbf{M} \pm S \\
D \\
\end{array}$ & $\begin{array}{c}\mathbf{1}^{\text {st }} \\
(\mathrm{M} \pm \mathrm{S} \\
\mathrm{D} \\
\end{array}$ & $\begin{array}{c}2^{\text {nd }} \\
(M \pm S \\
D \\
\end{array}$ & $\begin{array}{c}3^{\text {rd }} \\
(M \pm S \\
D \\
\end{array}$ & $\begin{array}{c}4^{\text {th }} \\
(M \pm S \\
D \\
\end{array}$ & $\begin{array}{c}\mathbf{5}^{\text {th }} \\
(\mathrm{M} \pm \mathrm{S} \\
\mathrm{D} \\
\end{array}$ & $\begin{array}{c}\text { 6 }^{\text {th }} \\
(\mathbf{M} \pm \mathbf{S} \\
\text { D } \\
\end{array}$ & $\begin{array}{c}7^{\text {th }} \\
(\mathrm{M} \pm \mathrm{S} \\
\mathrm{D} \\
\end{array}$ & $\begin{array}{c}\mathbf{8}^{\text {th }} \\
(\mathbf{M} \pm \mathbf{S} \\
D \\
\end{array}$ \\
\hline & Ad & $\begin{array}{l}1.33 \\
\pm \\
0.12 \\
\end{array}$ & $\begin{array}{l}2.13 \\
\pm \\
0.17 \\
\end{array}$ & $\begin{array}{l}2.93 \\
\pm \\
0.15\end{array}$ & $\begin{array}{l}3.70 \\
\pm \\
0.20 \\
\end{array}$ & $\begin{array}{l}4.50 \\
\pm \\
0.30 \\
\end{array}$ & $\begin{array}{l}5.77 \\
\pm \\
0.30 \\
\end{array}$ & $\begin{array}{l}7.10 \\
\pm \\
0.50 \\
\end{array}$ & $\begin{array}{l}8.16 \\
\pm \\
0.45 \\
\end{array}$ & $\begin{array}{l}1.38 \\
\pm \\
0.14 \\
\end{array}$ & $\begin{array}{l}2.15 \\
\pm \\
0.15 \\
\end{array}$ & $\begin{array}{l}2.91 \\
\pm \\
0.10 \\
\end{array}$ & $\begin{array}{l}4.20 \\
\pm \\
0.20 \\
\end{array}$ & $\begin{array}{l}4.57 \\
\pm \\
0.25\end{array}$ & $\begin{array}{l}5.68 \\
\pm \\
0.25 \\
\end{array}$ & $\begin{array}{l}7.00 \\
\pm \\
0.40 \\
\end{array}$ & $\begin{array}{l}8.18 \\
\pm \\
0.40 \\
\end{array}$ \\
\hline & Se. & $\begin{array}{l}1.26 \\
\pm \\
0.20 \\
\end{array}$ & $\begin{array}{l}2.03 \\
\pm \\
0.20 \\
\end{array}$ & $\begin{array}{l}2.87 \\
\pm \\
0.12\end{array}$ & $\begin{array}{l}4.43 \\
\pm \\
0.35 \\
\end{array}$ & $\begin{array}{l}4.67 \\
\pm \\
0.30 \\
\end{array}$ & $\begin{array}{l}6.00 \\
\pm \\
0.50 \\
\end{array}$ & $\begin{array}{l}7.0 \\
\pm \\
0.50 \\
\end{array}$ & $\begin{array}{l}8.07 \\
\pm \\
0.45 \\
\end{array}$ & $\begin{array}{l}1.28 \\
\pm \\
0.10 \\
\end{array}$ & $\begin{array}{l}2.10 \\
\pm \\
0.10 \\
\end{array}$ & $\begin{array}{l}2.80 \\
\pm \\
0.20 \\
\end{array}$ & $\begin{array}{l}3.67 \\
\pm \\
0.20 \\
\end{array}$ & $\begin{array}{l}4.40 \\
\pm \\
0.10\end{array}$ & $\begin{array}{l}5.40 \\
\pm \\
0.30\end{array}$ & $\begin{array}{l}6.93 \\
\pm \\
0.40 \\
\end{array}$ & $\begin{array}{l}8.10 \\
\pm \\
0.10 \\
\end{array}$ \\
\hline \multirow{3}{*}{$\begin{array}{l}\text { Cross- } \\
\text { fertiliz } \\
\text { ation }\end{array}$} & $\begin{array}{l}\text { Ad } \\
\& \\
\text { Ad }\end{array}$ & $\begin{array}{l}1.23 \\
\pm \\
0.15\end{array}$ & $\begin{array}{l}2.08 \\
\pm \\
0.20\end{array}$ & $\begin{array}{l}2.87 \\
\pm \\
0.15\end{array}$ & $\begin{array}{l}3.93 \\
\pm \\
0.15\end{array}$ & $\begin{array}{l}4.60 \\
\pm \\
0.15\end{array}$ & $\begin{array}{l}5.77 \\
\pm \\
0.25\end{array}$ & $\begin{array}{l}7.00 \\
\pm \\
0.35\end{array}$ & $\begin{array}{l}8.23 \\
\pm \\
0.30\end{array}$ & $\begin{array}{l}1.27 \\
\pm \\
0.10\end{array}$ & $\begin{array}{l}2.23 \\
\pm \\
0.16\end{array}$ & $\begin{array}{l}2.90 \\
\pm \\
0.25\end{array}$ & $\begin{array}{l}3.80 \\
\pm \\
0.15\end{array}$ & $\begin{array}{l}4.60 \\
\pm \\
0.20\end{array}$ & $\begin{array}{l}5.67 \\
\pm \\
0.25\end{array}$ & $\begin{array}{l}7.00 \\
\pm \\
0.35 \\
\end{array}$ & $\begin{array}{l}8.10 \\
\pm \\
0.40\end{array}$ \\
\hline & $\begin{array}{l}\text { Se } \\
\& \\
\text { Ad }\end{array}$ & $\begin{array}{l}1.23 \\
\pm \\
0.15 \\
\end{array}$ & $\begin{array}{l}2.70 \\
\pm \\
0.15\end{array}$ & $\begin{array}{l}2.83 \\
\pm \\
0.15\end{array}$ & $\begin{array}{l}3.80 \\
\pm \\
0.20 \\
\end{array}$ & $\begin{array}{l}4.53 \\
\pm \\
0.15 \\
\end{array}$ & $\begin{array}{l}5.73 \\
\pm \\
0.30 \\
\end{array}$ & $\begin{array}{l}7.00 \\
\pm \\
0.20\end{array}$ & $\begin{array}{l}8.20 \\
\pm \\
0.20 \\
\end{array}$ & $\begin{array}{l}1.17 \\
\pm \\
0.15\end{array}$ & $\begin{array}{l}2.88 \\
\pm \\
0.10\end{array}$ & $\begin{array}{l}2.93 \\
\pm \\
0.15 \\
\end{array}$ & $\begin{array}{l}3.70 \\
\pm \\
0.25 \\
\end{array}$ & $\begin{array}{l}4.57 \\
\pm \\
0.25 \\
\end{array}$ & $\begin{array}{l}4.70 \\
\pm \\
0.30\end{array}$ & $\begin{array}{l}7.03 \\
\pm \\
0.25 \\
\end{array}$ & $\begin{array}{l}8.00 \\
\pm \\
0.30 \\
\end{array}$ \\
\hline & $\begin{array}{l}\mathrm{Se} \\
\& \\
\mathrm{Se}\end{array}$ & $\begin{array}{l}1.21 \\
\pm \\
0.15\end{array}$ & $\begin{array}{l}2.03 \\
\pm \\
0.20\end{array}$ & $\begin{array}{l}2.83 \\
\pm \\
0.15\end{array}$ & $\begin{array}{l}3.83 \\
\pm \\
0.15\end{array}$ & $\begin{array}{l}4.53 \\
\pm \\
0.20\end{array}$ & $\begin{array}{l}5.67 \\
\pm \\
0.35\end{array}$ & $\begin{array}{l}6.99 \\
\pm \\
0.25\end{array}$ & $\begin{array}{l}8.20 \\
\pm \\
0.30\end{array}$ & $\begin{array}{l}1.20 \\
\pm \\
0.10\end{array}$ & $\begin{array}{l}2.17 \\
\pm \\
0.20\end{array}$ & $\begin{array}{l}2.90 \\
\pm \\
0.10\end{array}$ & $\begin{array}{l}3.77 \\
\pm \\
0.25\end{array}$ & $\begin{array}{l}4.56 \\
\pm \\
0.20\end{array}$ & $\begin{array}{l}5.60 \\
\pm \\
0.40\end{array}$ & $\begin{array}{l}6.97 \\
\pm \\
0.35\end{array}$ & $\begin{array}{l}8.00 \\
\pm \\
0.30\end{array}$ \\
\hline Anova & N.S. & N.S. & N.S. & N.S. & N.S. & N.S. & N.S. & N.S. & N.S. & N.S. & N.S. & N.S. & N.S. & N.S. & N.S. & N.S. & N.S. \\
\hline
\end{tabular}

N.S.: Non-significant

Table (8): Growth rate of neonatal offspring derived by cross-fertilization of different strains within the same snail species (either B. alexandrina or B. glabrata) at different ages.

\begin{tabular}{|c|c|c|c|c|c|c|c|c|c|c|c|c|c|c|c|c|}
\hline Snail & \multicolumn{16}{|c|}{ Time of Examination / week } \\
\hline \multirow{2}{*}{$\begin{array}{c}\text { phenoty } \\
\text { pe }\end{array}$} & \multicolumn{8}{|c|}{ B. alexandrina } & \multicolumn{8}{|c|}{ B. glabrata } \\
\hline & $\begin{array}{c}1^{\text {st }} \\
(\mathrm{M} \pm \mathrm{S} \\
\mathrm{D}) \\
\end{array}$ & $\begin{array}{c}2^{\text {nd }} \\
(M \pm S \\
D) \\
\end{array}$ & $\begin{array}{c}3^{\text {rd }} \\
(\mathbf{M} \pm \mathbf{S} \\
\mathrm{D}) \\
\end{array}$ & $\begin{array}{c}4^{\text {th }} \\
(M \pm S \\
D)\end{array}$ & $\begin{array}{c}5^{\text {th }} \\
(\mathbf{M} \pm \mathbf{S} \\
D)\end{array}$ & $\begin{array}{c}6^{\text {th }} \\
(M \pm S \\
D) \\
\end{array}$ & $\begin{array}{c}7^{\text {th }} \\
(M \pm S \\
D)\end{array}$ & $\begin{array}{c}8^{\text {th }} \\
(M \pm S \\
D)\end{array}$ & $\begin{array}{c}\mathbf{1}^{\text {st }} \\
(\mathbf{M} \pm \mathbf{S} \\
\mathrm{D})\end{array}$ & $\begin{array}{c}2^{\text {nd }} \\
(\mathbf{M} \pm \mathbf{S} \\
D) \\
\end{array}$ & $\begin{array}{c}3^{\text {rd }} \\
(M \pm S \\
D)\end{array}$ & $\begin{array}{c}4^{\text {th }} \\
(\mathrm{M} \pm \mathrm{S} \\
\mathrm{D})\end{array}$ & $\begin{array}{c}5^{\text {th }} \\
(M \pm S \\
\text { D) }\end{array}$ & $\begin{array}{c}\mathbf{6}^{\text {th }} \\
(\mathbf{M} \pm \mathbf{S} \\
\mathrm{D}) \\
\end{array}$ & $\begin{array}{c}7^{\text {th }} \\
(M \pm S \\
D)\end{array}$ & $\begin{array}{c}\mathbf{8}^{\text {th }} \\
(\mathrm{M} \pm \mathrm{S} \\
\mathrm{D}) \\
\end{array}$ \\
\hline $\begin{array}{l}\text { AdS\& } \\
\text { AdR }\end{array}$ & $\begin{array}{l}1.25 \\
\pm \\
0.13\end{array}$ & $\begin{array}{l}2.27 \\
\pm \\
0.10\end{array}$ & $\begin{array}{l}2.70 \\
\pm \\
0.10\end{array}$ & $\begin{array}{l}3.50 \\
\pm \\
0.15\end{array}$ & $\begin{array}{l}4.00 \\
\pm \\
0.15\end{array}$ & $\begin{array}{l}4.70 \\
\pm \\
0.30\end{array}$ & $\begin{array}{l}5.70 \\
\pm \\
0.30\end{array}$ & $\begin{array}{l}6.80 \\
\pm \\
0.30\end{array}$ & $\begin{array}{l}1.35 \\
\pm \\
0.13\end{array}$ & $\begin{array}{l}2.28 \\
\pm \\
0.10\end{array}$ & $\begin{array}{l}2.90 \\
\pm \\
0.10\end{array}$ & $\begin{array}{l}3.67 \\
\pm \\
0.15\end{array}$ & $\begin{array}{l}4.63 \\
\pm \\
0.15\end{array}$ & $\begin{array}{l}5.70 \\
\pm \\
0.30\end{array}$ & $\begin{array}{l}7.00 \\
\pm \\
0.30\end{array}$ & $\begin{array}{l}8.10 \\
\pm \\
0.20\end{array}$ \\
\hline $\begin{array}{ll}\mathrm{Ad}_{\mathrm{S}} & \& \\
\mathrm{Se}_{\mathrm{R}} & \end{array}$ & $\begin{array}{l}1.22 \\
\pm \\
0.15\end{array}$ & $\begin{array}{l}2.20 \\
\pm \\
0.20\end{array}$ & $\begin{array}{l}2.68 \\
\pm \\
0.15 \\
\end{array}$ & $\begin{array}{l}3.30 \\
\pm \\
0.20\end{array}$ & $\begin{array}{l}3.90 \\
\pm \\
0.10 \\
\end{array}$ & $\begin{array}{l}4.63 \\
\pm \\
0.15\end{array}$ & $\begin{array}{l}5.60 \\
\pm \\
0.30\end{array}$ & $\begin{array}{l}6.70 \\
\pm \\
0.30\end{array}$ & $\begin{array}{l}1.30 \\
\pm \\
0.10\end{array}$ & $\begin{array}{l}2.80 \\
\pm \\
0.15 \\
\end{array}$ & $\begin{array}{l}2.89 \\
\pm \\
0.10\end{array}$ & $\begin{array}{l}3.53 \\
\pm \\
0.15\end{array}$ & $\begin{array}{l}4.53 \\
\pm \\
0.15\end{array}$ & $\begin{array}{l}5.67 \\
\pm \\
0.25 \\
\end{array}$ & $\begin{array}{l}7.01 \\
\pm \\
0.25\end{array}$ & $\begin{array}{l}8.01 \\
\pm \\
0.20\end{array}$ \\
\hline $\begin{array}{l}\mathrm{Ad}_{\mathrm{R}} \quad \& \\
\mathrm{Se}_{\mathrm{S}}\end{array}$ & $\begin{array}{l}1.13 \\
\pm \\
0.15\end{array}$ & $\begin{array}{l}2.33 \\
\pm \\
0.20\end{array}$ & $\begin{array}{l}2.70 \\
\pm \\
0.10\end{array}$ & $\begin{array}{l}3.40 \\
\pm \\
0.10\end{array}$ & $\begin{array}{l}3.90 \\
\pm \\
0.10\end{array}$ & $\begin{array}{l}4.63 \\
\pm \\
0.20\end{array}$ & $\begin{array}{l}5.67 \\
\pm \\
0.25\end{array}$ & $\begin{array}{l}6.73 \\
\pm 0.25\end{array}$ & $\begin{array}{l}1.28 \\
\pm \\
0.10\end{array}$ & $\begin{array}{l}2.85 \\
\pm \\
0.20\end{array}$ & $\begin{array}{l}2.90 \\
\pm \\
0.10\end{array}$ & $\begin{array}{l}3.60 \\
\pm \\
0.10\end{array}$ & $\begin{array}{l}4.57 \\
\pm \\
0.20\end{array}$ & $\begin{array}{l}5.60 \\
\pm \\
0.30\end{array}$ & $\begin{array}{l}7.02 \\
\pm 0.25\end{array}$ & $\begin{array}{l}8.03 \\
\pm \\
0.15\end{array}$ \\
\hline $\begin{array}{ll}\mathrm{SeS} & \& \\
\mathrm{SeR} & \end{array}$ & $\begin{array}{l}1.10 \\
\pm \\
0.09\end{array}$ & $\begin{array}{l}2.20 \\
\pm \\
0.20\end{array}$ & $\begin{array}{l}2.70 \\
\pm \\
0.15\end{array}$ & $\begin{array}{l}3.40 \\
\pm \\
0.10\end{array}$ & $\begin{array}{l}3.90 \\
\pm \\
0.10\end{array}$ & $\begin{array}{l}4.65 \\
\pm \\
0.27\end{array}$ & $\begin{array}{l}5.63 \\
\pm \\
0.30\end{array}$ & $\begin{array}{l}6.70 \\
\pm \\
0.30\end{array}$ & $\begin{array}{l}1.25 \\
\pm \\
0.20\end{array}$ & $\begin{array}{l}2.13 \\
\pm \\
0.10\end{array}$ & $\begin{array}{l}2.85 \\
\pm \\
0.15\end{array}$ & $\begin{array}{l}3.83 \\
\pm \\
0.15\end{array}$ & $\begin{array}{l}4.53 \\
\pm \\
0.30\end{array}$ & $\begin{array}{l}5.57 \\
\pm \\
0.25\end{array}$ & $\begin{array}{l}6.98 \\
\pm \\
0.30\end{array}$ & $\begin{array}{l}8.30 \\
\pm \\
0.15\end{array}$ \\
\hline Anova & N.S. & N.S. & N.S. & N.S. & N.S. & N.S. & N.S. & N.S. & N.S. & N.S. & N.S. & N.S. & N.S. & N.S. & N.S. & N.S. \\
\hline
\end{tabular}

N.S.: Non-significant 


\section{B. alexandrina}

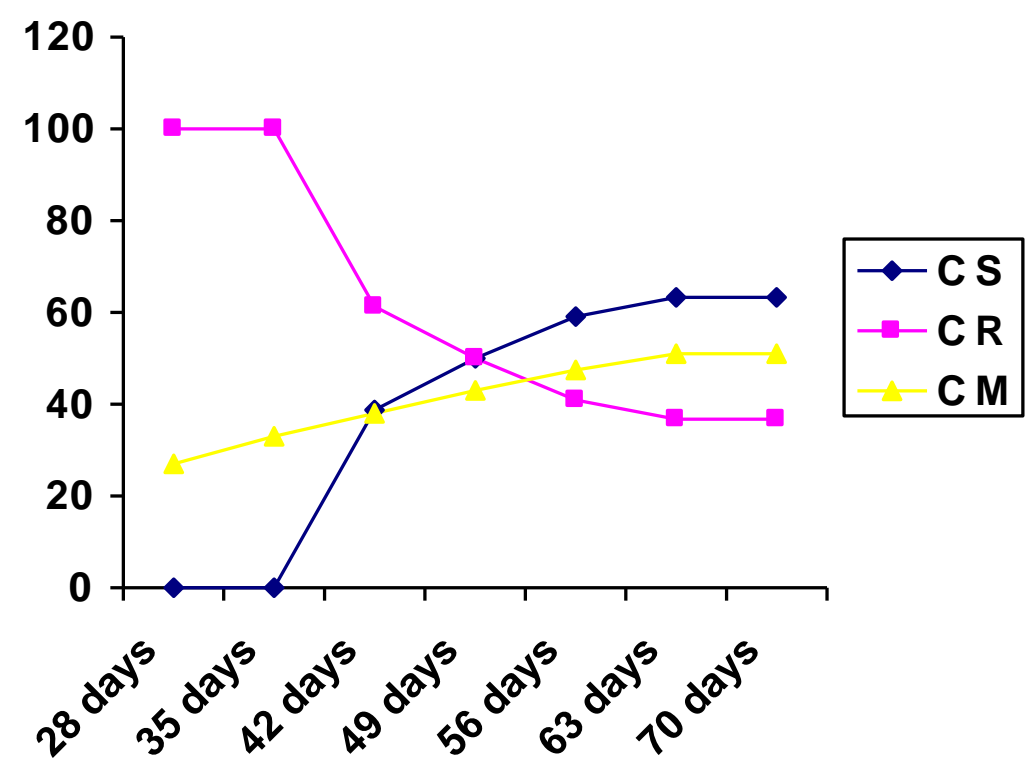

B. glabrata

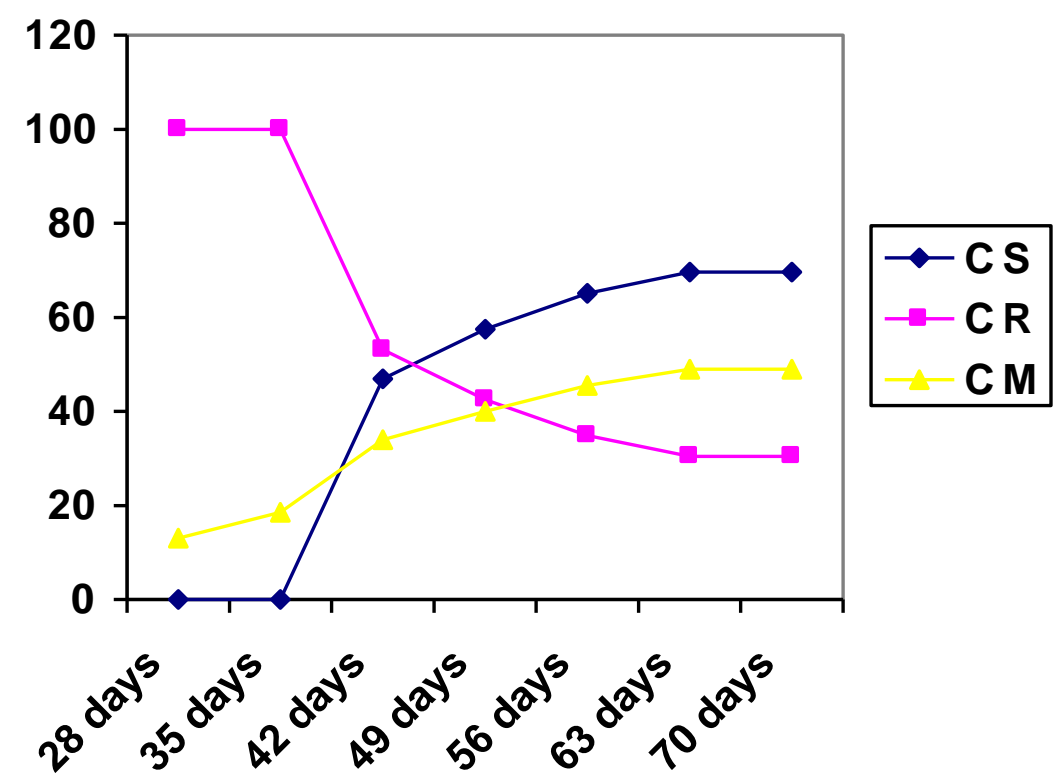

CS. : Cumulative percentage of susceptible strain

CR: Cumulative percentage of resistant strain .

$\mathrm{CM}$ : Cumulative percentage of mortality rate .

Fig (1): Cumulative susceptibility / resistance and mortality rate percentage in $B$. alexandrina or B. glabrata after miracidial expousure . 


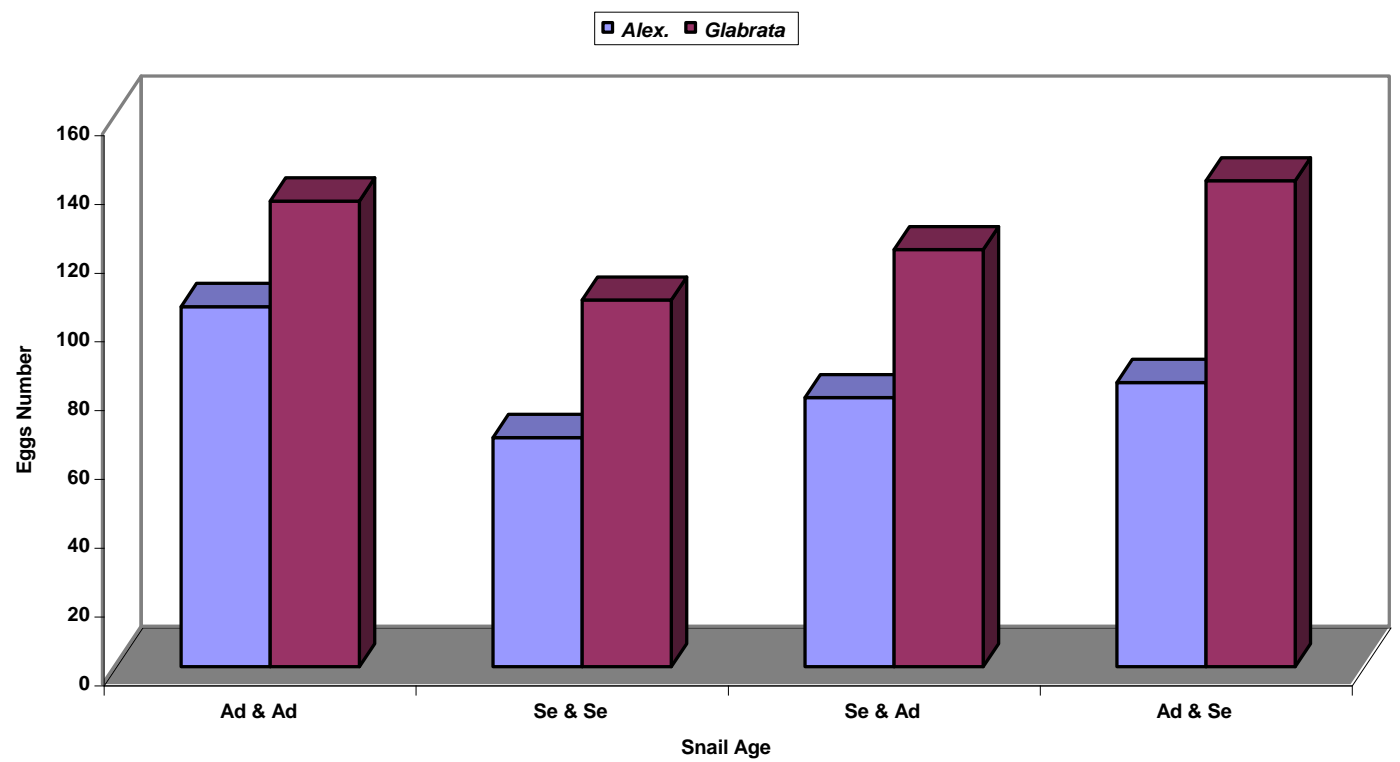

Fig ( 2 ): Total egg number produced by cross fertilization between susceptible and resistant strain of both $B$. alexandrina and B. glabrata at different ages.

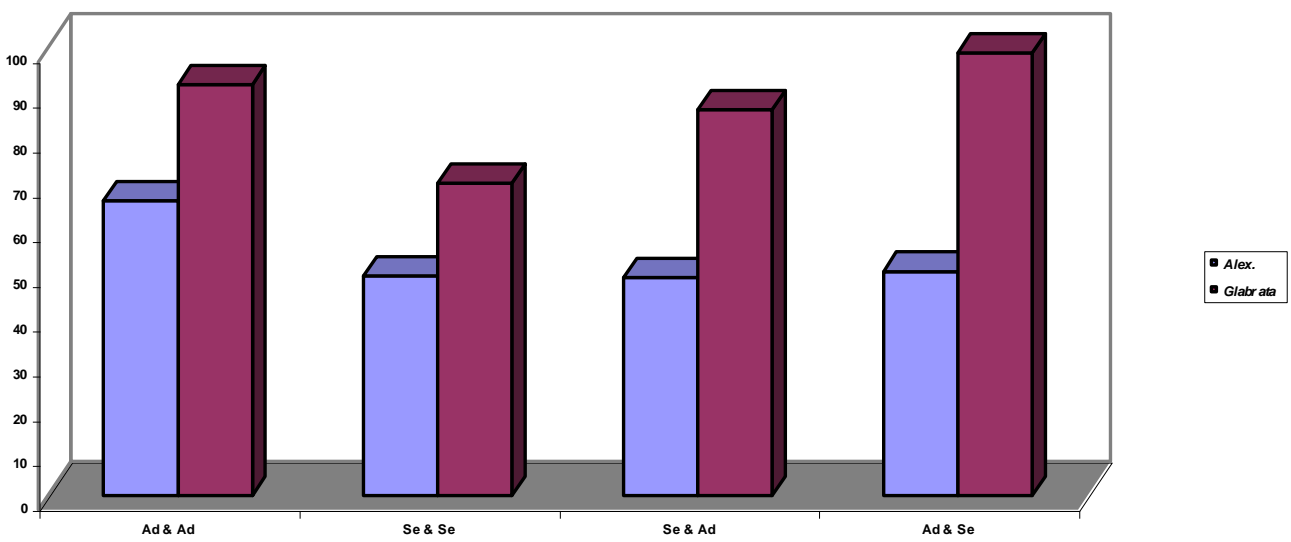

Fig ( 3 ): Cumulative number of hatching snails produced by cross fertilization between susceptible and resistant strain of both $B$. alexandrina and $B$. glabrata at different ages .

\section{Discussion}

It is well acknowledged that the distribution of schistosomiasis is focal ( Sturrock, 1993 ), and that transmission only takes place in area where intermediate host snails are present and where the human population is frequently in contact with infected water.

The results of the present study obtained by experimental infection with a high dose of 10 miracidia exhibits $36.7 \%$ and $30.4 \%$ of both $B$. alexandrina and $B$. glabrata are resistant to $S$. mansoni infection comparing with $63.3 \%$ and 69.6 $\%$ of their snails which yielded high infection frequencies, respectively. These results confirmed that $B$. alexandrina and B. glabrata are important schistosomiasis vector in Egypt . Moreover, the finding of 
Yousif et al. ( 1996 ) gave some support to this suggestion, by reporting that $B$. glabrata forms a new threat for schistosomiasis transmission in Egypt. The collected B. glabrata snails from natural water courses and proved them to be susceptible to a local strain of $S$. mansoni with infection rates $52 \%$ versus $75 \%$ for $B$. alexandrina under the same laboratory conditions. In the same respect, Haroun ( 1996 ) examined 53.9 - $60 \%$ infection rate of $B$. alexandrina. Similar results were recorded by Spada et al. ( 2002 ) who examined $63 \%$ of $B$. glabrata snails yielded high infection frequencies in contrast to $37 \%$ of these snails were refractory .

In the current study, the variability in susceptibility / resistance of Biomphalaria species to Schistosoma infection are in agreement with the results obtained by Souza et al. (1995 ) who demonstrated considerable variations in susceptibility in different Biomphalaria species . Such variability can even be observed in snails belonging to the same species, from different geographical areas ( Richards , 1984 ). Thus, resistance or susceptibility is genetically dependent rather than acquired immunity ( Richards , 1976 ) which may ultimately be related to genetic background ( Lemos and Andrade, 2001 ).

Although both $B$. alexandrina and $B$. glabrata snails are hermaphroditic and can self-fertilize, the obtained results support the phenomenon that the snails prefer to reproduce by cross-fertilization when paired. These biological characteristics are evolutionary very significant, providing the snail with the ability to establish or reestablish colonies from an individual organism with maintaining the genetic characters through sexual reproduction .

A number of molluscan intermediate host species is hermaphrodite and can self fertilize but prefer to reproduce by crossfertilization when paired ( Vianey-Liaud and Dussart , 2002 ; Trouve et al., 2003 ) . The present study was further extended to compare the mean frequencies of egg production as reflected by EM , hatching offspring and hatchlings reaching maturity among progeny snail groups of susceptible and resistant Biomphalaria snails either by self-or cross- fertilization at different ages .

The present results indicated that the level of egg production in any given group of both susceptible and resistant either selfed or crossed snail stocks at different ages ranged from 216 - 256 egg / month / snail and from $322-412$ egg / month / snail of both $B$. alexandrina and $B$. glabrata, respectively. Such results agree with the results obtained by Lewis et al. ( 1986 ) who demonstrated that a single $B$. glabrata snail can produce $200-500$ eggs / month. They also reported that $50-100$ egg-laying snails should provide more than enough eggs to meet the demands of a large life cycle production .

In the present study, the patterns of oviposition showed a high significant reduction in the actual reproductive output of susceptible snails, within fewer embryos being produced per week than resistant ones. These results agree with previous results obtained by some authors who demonstrated that, upon infection ( generally in susceptible snails) of schistosome with their molluscan hosts, reproduction is reduced but never completely repressed (Schrag and Rollinson, 1994 ; Jong-Brink , 1995 ; Cooper et al., 1996 ; Johston et al ., 1997 ).

During the course of studies on the reproductive biology of $B$. alexandrina and $B$. glabrata, the mean number of eggs produced by cross-fertilization was significantly higher than those corresponding in self-fertilization. Similar results were obtained by Vernon ( 1995 ), who found that, isolated self-fertilizing individuals of B. glabrata had reduced reproductive output compared with paired predominantly cross-fertilizing snails. However, the reproductive output of control snails ( kept in pairs, but prevented from crossfertilizing ) was similar to that of paired cross-fertilizing snails, suggesting that the low reproductive output of snails in isolation was not simply due to inbreeding depression with self-fertilization .

It has been demonstrated from the present results that, the egg masses produced from an inbreeding of both susceptible of either self or cross- 
fertilization develop and hatch normally with many embryos per-clutches, but with lower rate than in resistant ones. These results are in agreement with the finding of Etges and Gresso ( 1965 ).

In current study, the number and percentage of newly haching snails and snail reaching maturity per selfing is lower than those of crossing and slightly higher in resistant strain than in susceptible ones . These observations tend to confirm those of Cooper et al.( 1994 ) on the effect of self and cross fertilization on the egg laying capacity of molluscan host , B. glabrata, as well as De Freitase et al. ( 1997 ), who reported that, the production of viable embryos by cross - fertilization was more than by self - fertilization performance .

Maturity was determined by onest of egg laying in our laboratory-bred Biomphalaria snails of F1 progeny at shell diameter approximately $5-7 \mathrm{~mm}$., but the growth rate in $B$. glabrata was faster than $B$. alexandrina. This result was supported by Malek ( 1958 ) who reported that, $B$. glabrata shell diameter ranged between 15 to $30 \mathrm{~mm}$. . On the other hand, $B$. alexandrina was reported to be maximally $19 \mathrm{~mm}$. in diameter by Meier and Meier Brook ( 1981 ).

Considering the results by age of snails and its effect on the output of reproductive system, it was shown that egg production is inversely proportional to the snail age and significantly higher in adult parental snails than in senescent ones. Similar results were obtained by Richards and Meritt ( 1972 ) which indicated that the onest of egg laying has been observed in B. glabrata around 5 $\mathrm{mm}$. in diameter .

\section{References}

1. Ayad N. (1974 ) : A short review of the epidemiology of schistosomiasis in Africa, Egypt. J. Bilh., 1: 9-27.

2. Bayomy MFF. and Joosse J. (1987) : The effect of isolation, grouping and population density on fecundity of Bulinus truncates. Int. J. Invertebr. Reprod. Dev., 12: 319-330.

3. Coelho JR. and Bezerra FSM. ( 2006 ) : The effects of temperature change on the infection rate of Biomphalaria glabrata with
Schistosoma mansoni . Mem Inst. Oswaldo Cruz Rio de Janeiro, $101: 223$ - 224 .

4. Cooper LA, Larson SE. and Lewis FA. (1996): Male reproductive success of Schistosoma mansoni infected Biomphalaria glabrata snails. J. Parasitol., 82 : 428-431.

5. Cooper LA, Richards CS, Lewis FA. and Minchella DJ. (1994): Schistosoma mansoni , relationship between low fecundity and reduced susceptibility to parasite infection in the snail Biomphalaria glabrata. Exp. Parasitol., 79: 21-28.

6. De Freitas JS, Paula DP. and Cariello MO. (1997): The influence of selffertilization performance and copulation behaviour in reproduction by crossfertilization in groups of Biomphalaria tenagophila (Mollusca : Planorbidae). Mem. Inst. Oswaldo. Cruz., 92 : 739-743.

7. Etges FJ. and Gresso W. (1965): Effect of Schistosoma mansoni infection upon fecundity in Australorbis glabratus. J. Parasitol., 51: 757-760.

8. Gibodat M. and Bergquist NR. ( 2000 ): Post - transmission Schistosomiasis : a new agenda . Acta Tropica , 77 pp. 3-7

9. Haroun NH. (1996): Differences in susceptibility of Biomphalaria alexandrina to Schistosoma mansoni from Giza and Dakahlia Govermorates, Egypt. J. Egypt. Soc. Parasitol., 26 : 327-335.

10. Jarne P. and Stadler T. (1995): Population genetic structure and mating system evolution in freshwater pulmonates. Experientia ., 51: 482-497.

11. Johston BG, Watson CO. and Yoshino TP. (1997): Phenoloxidase activity in the reproductive system of Biomphalaria glabrata role in egg production and effect of schistosome infection. J. Parasitol., 83 : 852858.

12. Jong-Brink M. (1995): How schistosomes profit from the stress responses they elicit in their hosts. Advanc. Parasitol., 35 : 177-256.

13. Langand L. and Morand S. ( 1998 ): Heritable non-susceptibility in an allopatric host-parasite system : Biomphalaria glabrata ( Mollusca ) Eschinostoma caproni ( platyhelminth Digenea ). J. Parasitol., 84 : $739-742$.

14. Larson SE, Anderson PL, Miller AN, Cousin CE, Richards CS, Lewis FA. and Knight M. (1996): Use of RAPD-PCR to differentiate genetically defined lines of an intermediate host of Schistosoma mansoni, Biomphalaria glabrata. J. Parasitol., 82 : 237-244. 
15. Lemos QT. and Andrade ZA. ( 2001 ): Sequential histological changes in Biomphalaria glabrata during the course of Schistosoma mansoni infection . Mem. Inst. Oswaldo . Cruz.,96: $719-721$.

16. Lewis FA, Stirewalt MA, Souza CP. and Gazzinelli G. (1986): Large-scale laboratory maintenance of Schistosoma mansoni, with observations on three schistosome snail host interactions. J. Parasitol., 72: 813-829.

17. Malek EA. (1958): factors conditioning the habitat of bilharziasis intermediate hosts of the family Planorbidae. Bull. WHO, 18 : 785-818.

18. Meier M. and Meier-Brook C. (1981): Schistosoma mansoni: Effect on growth, fertility, and development of distal male organs in Biomphalaria glabrata exposed to miracidia at different ages. Z. Parasitenkd., 66: 121-131.

19. Pitchford RJ. (1981): Temperature and schistosome distribution in south Africa. South African Journal of Science , 77: 252267.

20. Richards CS. ( 1976 ) : Variations in infectivity for Biomphalaria glabrata in strains of Schistosoma mansoni from the same geographic area . Bull. WHO, 54 : 706 -707 .

21. Richards CS. (1984): Influence of snail age on genetic variations in susceptibility of Biomphalaria glabrata for infection with Schistosoma mansoni. Malacology., 25: 493-502.

22. Richards CS. and Meritt JWJ. ( 1972 ) : Genetic factors in the susceptibility of juvenile Biomphalaria glabrata of Schistosoma mansoni infection. Am. J. Trop. Med. Hyg., $21: 425-435$.

23. Schrag SJ. and Rollinson D. (1994): Effect of Schistosoma haematobium infection on reproductive success and male outcrossing ability in the simultaneous hermaphrodite, Bulinus truncatus (gastropoda: Planorbidae). Parasitology., 108: 27-34.

24. Sousa RP, Janotti - Passos LK. and Freitase JR. ( 1995 ): Degree of hostparasite compatibility between Schistosoma mansoni and their intermediate molluscan host in Brazil . Mem. Inst. Oswaldo - Cruz., $90: 5-10$

25. Spada RG, Da Silva D, Abdel-Hamid AZ, Sobral-Hamaguchi SS, Zuim NR, Zanotti-Magalhaes EM, Magalhaes LA. and Ribeiro-Paes JT. (2002): Genetic markers between Biomphalaria glabrata snails susceptible and resistant to Schistosoma mansoni infection. Mem. Inst. Oswaldo. Cruz., 97 : 53-58.

26. Sturrock RF. (1993): The intermediate hosts and host-parasite relationships. Human Shistosomiasis ., 33-85.

27. Trouve S, Degen L, Renaud F. and Goudet J. (2003): Evolutionary implications of a high selfing rate in the freshwater snail lymnaea truncatula. Evolution, 57 : 2303-2314.

28. Vernon JG. (1995): Low reproductive output of isolated, self-fertilizing snails: inbreeding depression or absence of social facilitation? Proc. R. Soc. Lond. B. 259: 131-136.

29. Vianey-Liaud M. and Dussart G. ( 2002 ): Aspects of pairing and reproduction in the hermaphrodite freshwater snail Biomphalaria glabrata (Gastropoda: Pulmonata). J. Molluscan Studies, 68 : 243-248.

30. Vidigal TH, Dias NE, Spatz L, Nunes DN, Pires ER, Simpson AJ. and Carvalho OS. (1998): Genetic variability and identification of the intermediate snail hosts of Schistosoma mansoni. Mem. Inst. Oswaldo. Cruz., 93: 103-110.

31. WHO( 2004 ): Schistosomiasis .

32. Yousif F, Haroun N, Ibrahim A. and ElBardicy S. (1996): Biomphalaria glabrata: A new threat for schistosomiasis transmission in Egypt. J. Egypt. Soc. Parasitol., $26: 191-205$.

33. Zanotti-Magalhaes EM, Magalhaes LA. and Carvalho JR. (1997): Relacao rntre pathogencidade do Schistosoma mansoni em camundongos e a susceptibilidade do molusco vector. IV. Infecciosidade dos miracidios. Rev. Saude Puplica, 29: 265-270 cited from Abdel-Hamid et al. (2005).

34. Zar JH. ( 1996 ): Biostatistical Analysis, $3^{\text {rd }}$ ed. , Prentica - Hall, New Jersey, 662 pp. 


\section{دراسات بيولوجية على قواقع الماء العذب المستهدفة لطفيلى البلهارسيا}

\section{عبد الحميد زكي عبد الحميد ، سامية محمد عبد الوهاب* ، منى محمد التونسي* ، رحاب ممدوح عبد المجيد}

من قسم الكيمباء العلاجية بالمركز القومي للبحوث وقدم علدم علم الحيوان بكلية علوم

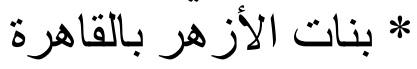

تلعب قو اقع المـاء العذب دوراً حيوياً في إنتشـار مرض البلهارسيا حيث أنها تمثل العائل

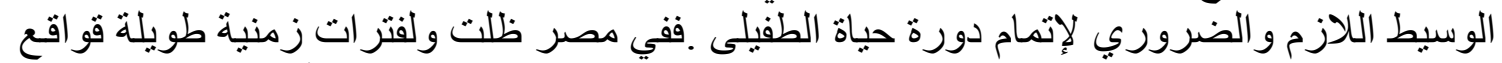

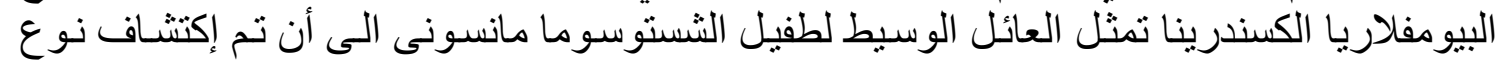

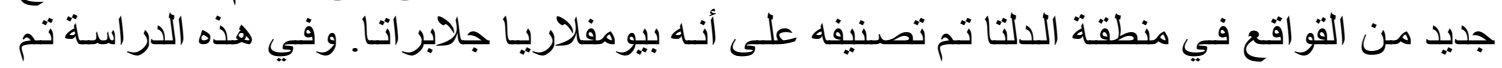

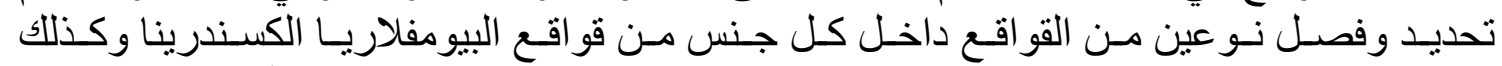

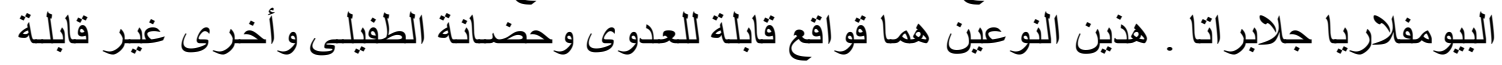

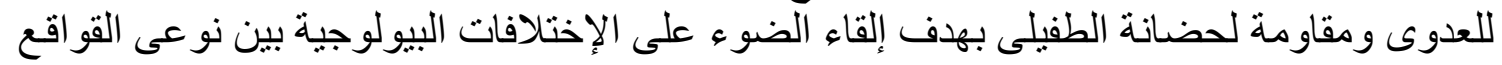

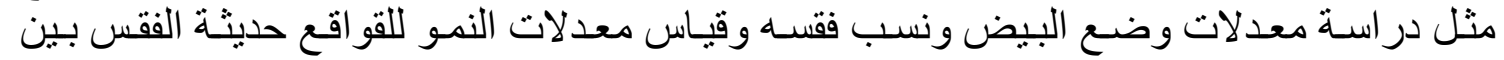

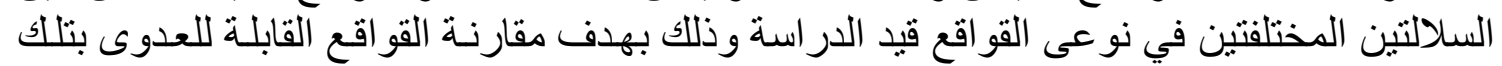

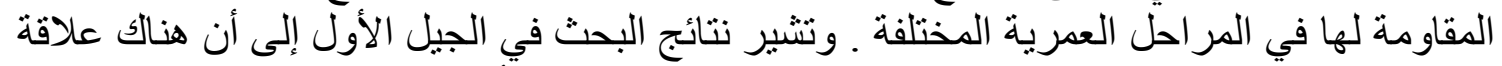

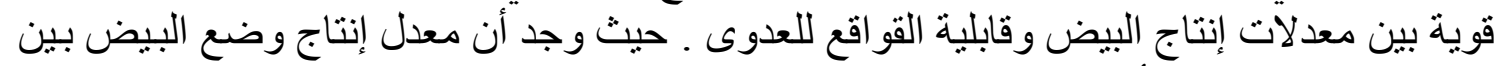

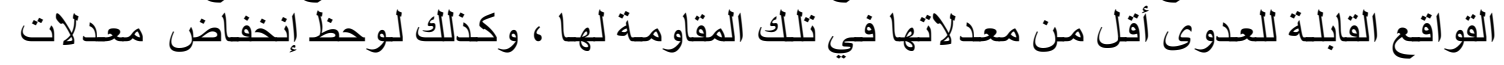

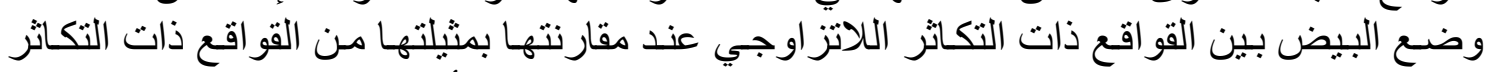

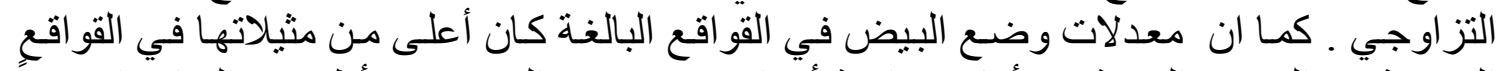

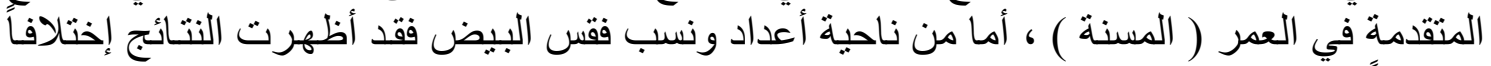

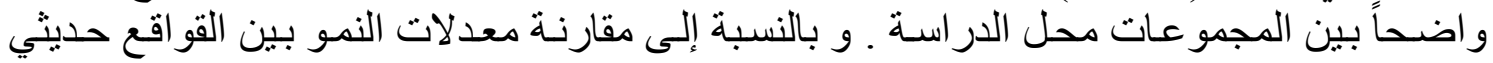

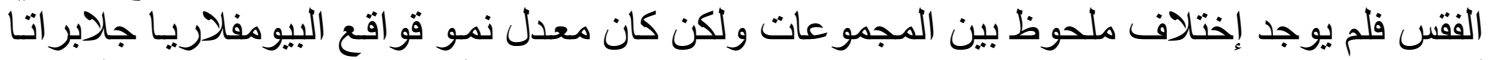

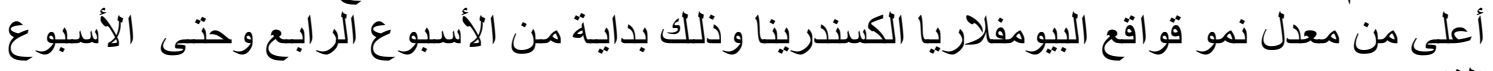

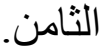

